\title{
The effect of Mindfulness-Based Stress Reduction on wound healing: a preliminary study
}

\author{
Astrid Meesters ${ }^{1}$ (D) - Yvo M. C. In den Bosch-Meevissen ${ }^{1}$ - Chantal A. H. Weijzen ${ }^{2}$ • \\ Wim A. Buurman ${ }^{3}$ Mario Losen ${ }^{3} \cdot$ Jan Schepers $^{4} \cdot$ Monique R. T. M. Thissen $^{5,6}$. \\ Hugo J. E. M. Alberts ${ }^{1}$ - Casper G. Schalkwijk ${ }^{7}$ Madelon L. Peters ${ }^{1}$
}

Received: June 28, 2017 / Accepted: November 6, 2017/Published online: November 20, 2017

(c) The Author(s) 2017. This article is an open access publication

\begin{abstract}
Psychological factors have been shown to influence the process of wound healing. This study examined the effect of Mindfulness-Based Stress Reduction (MBSR) on the speed of wound healing. The local production of pro-inflammatory cytokines and growth factors was studied as potential underlying mechanism. Forty-nine adults were randomly allocated to a waiting-list control group $(\mathrm{n}=26)$ or an 8-week MBSR group $(\mathrm{n}=23)$. Preand post-intervention/waiting period assessment for both groups consisted of questionnaires. Standardized skin
\end{abstract}

Electronic supplementary material The online version of this article (https://doi.org/10.1007/s10865-017-9901-8) contains supplementary material, which is available to authorized users.

Astrid Meesters

astrid.meesters@maastrichtuniversity.nl

Yvo M. C. In den Bosch-Meevissen

yvoindenbosch@gmail.com

Chantal A. H. Weijzen

c.weijzen@student.maastrichtuniversity.nl

Wim A. Buurman

w.buurman@maastrichtuniversity.nl

Mario Losen

m.losen@maastrichtuniversity.nl

Jan Schepers

jan.schepers@maastrichtuniversity.nl

Monique R. T. M. Thissen

monique.thissen@mumc.nl

Hugo J. E. M. Alberts

h.alberts@maastrichtuniversity.nl

Casper G. Schalkwijk

c.schalkwijk@maastrichtuniversity.nl

Madelon L. Peters

madelon.peters@maastrichtuniversity.nl wounds were induced on the forearm using a suction blister method. Primary outcomes were skin permeability and reduction in wound size monitored once a day at day 3,4 , 5, 6, 7, and 10 after injury. Secondary outcomes were cytokines and growth factors and were measured in wound exudates obtained at 3,6 , and $22 \mathrm{~h}$ after wounding. Although there was no overall condition effect on skin permeability or wound size, post hoc analyses indicated that larger increases in mindfulness were related to greater reductions in skin permeability 3 and 4 days after wound induction. In addition, MBSR was associated with lower levels of interleukin (IL)-8 and placental growth factor in the wound fluid $22 \mathrm{~h}$ after wound induction. These outcomes suggest that increasing mindfulness by MBSR might

1 Department of Clinical Psychological Science, Faculty of Psychology and Neuroscience, Maastricht University, P.O. Box 616, 6200 MD Maastricht, The Netherlands

2 Department of Family Medicine, Faculty of Health, Medicine and Life Sciences, Maastricht University, P.O. Box 616, 6200 MD Maastricht, The Netherlands

3 Department of Psychiatry and Neuropsychology, Faculty of Health, Medicine and Life Sciences, Maastricht University, P.O. Box 616, 6200 MD Maastricht, The Netherlands

4 Department of Methodology and Statistics, Faculty of Psychology and Neuroscience, Maastricht University, P.O. Box 616, 6200 MD Maastricht, The Netherlands

5 Department of Dermatology, Maastricht University Medical Centre+, P. Debyelaan 25, 6202 AZ Maastricht, The Netherlands

6 GROW School for Oncology and Developmental Biology, Maastricht University Medical Centre+, Universiteitssingel 40, P.O. Box 616, 6200 MD Maastricht, The Netherlands

7 Department of Internal Medicine, Faculty of Health, Medicine and Life Sciences, Maastricht University, P.O. Box 616, 6200 MD Maastricht, The Netherlands 
have beneficial effects on early stages of wound healing. Trial Registration NTR3652, http://www.trialregister.nl

Keywords Wound healing - Mindfulness - Intervention · Inflammation · Cytokine $\cdot$ Growth factor

\section{Introduction}

Over the past decades studies have repeatedly shown that psychological factors can influence the process of wound healing (e.g. Broadbent and Koschwanez 2012; House 2015). Most of these studies focused on the effects of psychosocial distress on wound healing and consistently reported impaired wound healing across different wound models and different stressors (Bosch et al. 2007; ColeKing and Harding 2001; Ebrecht et al. 2004; Garg et al. 2001; Kiecolt-Glaser et al. 2005; Maple et al. 2015). A meta-analysis revealed a robust association between stress and impaired healing (Walburn et al. 2009). The effects of stress on wound healing can be mediated by multiple immune and neuroendocrine pathways and may affect various stages of the healing process (Christian et al. 2006; Gouin and Kiecolt-Glaser 2011).

Pro-inflammatory cytokines such as interleukin (IL)- $1 \alpha$, IL-1 $\beta$, IL-6, IL-8 and tumor necrosis factor (TNF)- $\alpha$ are crucial during the early phases of the healing process, and thus for successful resolution of wounds. Their function is to remove damaged tissue, limit infection and to stimulate tissue repair (Lowry 1993). Consequently, upregulation of pro-inflammatory cytokines at the wound site is seen during the inflammatory phase of healing (Werner and Grose 2003). A potential mechanism responsible for the effect of stress on the healing process may consist of the suppression of pro-inflammatory cytokine activity at the wound site. Using an experimental wound induction procedure, Glaser et al. (1999) and Kiecolt-Glaser et al. (2005) have found lower levels in wound fluid of respectively the pro-inflammatory cytokines IL- $1 \alpha$ and IL-8 at 5 and $24 \mathrm{~h}$ and IL6 , IL-1 $\beta$, and TNF- $\alpha$ at $22 \mathrm{~h}$ after wound induction in participants reporting high stress levels or after stress induction. Broadbent et al. (2003) studied surgical wounds and found that pre-operative stress was related to lower levels of IL-1 $\beta$ in the wound fluid. However, there may not be a simple linear association between the expression of pro-inflammatory cytokines and the healing process. Adequate wound healing depends on the delicate balance between pro- and anti-inflammatory effects. Excessive inflammation delays wound healing; indeed higher concentrations of pro-inflammatory cytokines have been found in chronic wounds (Beidler et al. 2009).

In addition to cytokines, growth factors such as vascular endothelial growth factor (VEGF) and placental growth factor (PlGF) are important for wound repair (Failla et al. 2000; Johnson and Wilgus 2014). The role of VEGF in particular is well documented and considered important in stimulating the formation of new blood vessels (i.e. angiogenesis), which is imperative for tissue restoration (Johnson and Wilgus 2014). Animal and human studies have shown that low levels of VEGF are related to impaired healing (Johnson and Wilgus 2014). Stress could delay wound healing through decreasing growth factors at the wound site. Glucocorticoid up-regulation has been shown to negatively affect angiogenesis by down-regulating VEGF expression in vitro (Nauck et al. 1998). Moreover, preliminary evidence exists demonstrating a stressinduced delay in wound healing accompanied by decreased VEGF expression in an animal model of wound healing (Pyter et al. 2014).

If stress can negatively affect wound healing, then reducing stress may have positive effects on the speed and quality of wound healing. Psychological interventions such as relaxation and disclosure writing have indeed been found to improve healing of surgical wounds (Broadbent et al. 2012), wounds induced by skin biopsy (Robinson et al. 2016; Weinman et al. 2008), and to speed recovery of skin permeability after mild injury induced by tape-stripping (Robinson et al. 2015).

Mindfulness-Based Stress Reduction (MBSR) is another intervention that might positively affect wound healing. MBSR is a type of meditation practice that is directed at promoting a controlled, nonjudgmental, and non-evaluative awareness of moment-to-moment experiences (Kabat-Zinn 1990). A recent meta-analysis showed that MBSR can effectively reduce stress, anxiety and depression and enhance quality of life in healthy individuals (Khoury et al. 2015). Rosenkranz et al. (2013) examined the effects of MBSR on the level of IL- 8 and TNF- $\alpha$ in skin blisters in response to a laboratory stressor and capsaicin exposure. No main effect of the intervention was found but more intensive MBSR practice was associated with a decrease in TNF- $\alpha$ level from pre- to post-training in response to psychosocial stress and an inflammatory stimulus.

The current study examined the effect of an 8-week MBSR intervention on the healing of blister skin wounds in healthy adults. Participants were not specifically selected for elevated stress levels to ensure comparability with previous studies investigating the effects of psychological interventions on wound healing in healthy participants (e.g. Koschwanez et al. 2013; Robinson et al. 2015, 2016; Weinman et al. 2008). In addition, MBSR has been found to reduce stress in healthy populations not selected for elevated stress levels (Khoury et al. 2015). Trans-epidermal water loss (TEWL) —an index for skin permeabilityand wound size were the primary outcomes and assessed until 10 days after injury. Secondary outcomes were pro- 
inflammatory cytokines IL- $1 \beta$, IL-6, IL- 8 and TNF- $\alpha$, the growth factors VEGF, PIGF and the soluble Fms-like tyrosine kinase-1 (sFlt-1) and were measured at 3,6 and $22 \mathrm{~h}$ post injury. We hypothesized that individuals receiving the MBSR training would display a faster decrease in TEWL and wound size over the course of 10 days. In subsequent exploratory analyses, we examined the effects of MBSR on pro-inflammatory cytokine and growth factor levels in wound fluid.

\section{Materials and methods}

\section{Participants}

Participants were 49 healthy adults aged $19-28$ years (mean age $22.1 \pm 2.1$ years; 8 men) recruited through pamphlets and posters at Maastricht University and Maastricht University Medical Centre. They were rewarded with a MBSR training and an additional financial compensation of 100 euro. Individuals were screened over the telephone followed by a dermatologist's visit. They were excluded if they documented health problems (e.g. diabetes mellitus, cancer, auto-immune diseases, hypo/hyperthyroidism, etc.) and/or medication use (e.g. psychotropic drugs, blood pressure regulators, immunosuppressive drugs, steroids, etc.). Also excluded were individuals who: smoked; consumed more than 10 caffeinated foods or drinks per day; consumed more than 20 (men) or 10 (women) alcoholic drinks per week; had a body mass index $\leq 18$ or $\geq 30$; experienced intensely stressful events in the last 3 months; received psychological treatment within the last 6 months; or reported experience with meditation practice. Additional exclusion criteria included having a blood and/or needle phobia, previous allergic responses to adhesives, bandages, tapes, or silicones, or dermal dysfunctions such as vitiligo, psoriasis, atopic dermatitis, and multiple moles. Written informed consent was obtained from all individual participants included in this study. The study was approved by the Medical Ethical Committee of Maastricht University Medical Centre.

\section{Measures}

\section{Questionnaires}

The Kentucky Inventory of Mindfulness Skills (KIMS) was used as manipulation check for the mindfulness intervention (Baer et al. 2004). This instrument measures four mindfulness skills: observing, describing, acting with awareness, and accepting without judgment. Internal consistency of the KIMS in the current sample was $\alpha=.82$ at pre- and $\alpha=.88$ at post-intervention. Additional ques- tionnaires were administered to measure the effects of the mindfulness intervention on dispositional mindfulness (Mindfulness Attention Awareness Scale (MAAS; Brown and Ryan 2003)), dispositional optimism (the revised Life Orientation Test (LOT-R; Scheier et al. 1994)), and rumination and reflection (the Rumination-Reflection Questionnaire (RRQ; Trapnell and Campbell 1999)). Cronbach's alpha for the MAAS in this sample was .85 and .80 at pre- and post-intervention, respectively. Similarly, the internal consistency of the LOT-R was good at both pre- $(\alpha=.82)$ and post-intervention $(\alpha=.89)$. Lastly, in the current sample, Cronbach's alpha was .89 at both preand post-intervention for the rumination subscale and .90 at pre- and .93 at post-intervention for the reflection subscale. Pre- and post-intervention questionnaire data were not available for 3 and 5 participants, respectively.

\section{Intervention}

Participants were randomly assigned to either a waiting-list control group (WLC; $\mathrm{n}=26 ; 5$ men) or a MBSR group $(\mathrm{n}=23 ; 3$ men $)$. The MBSR intervention followed a standardized 8-week MBSR protocol (Kabat-Zinn 1982) with some elements from Mindfulness-Based Cognitive Therapy (Segal et al. 2002) taught by an experienced trainer. Two successive groups of 15 people each were conducted. Three individuals in the MBSR group received advice from the dermatologist not to participate in the wound healing part of the study and were therefore excluded. Another four individuals were excluded because they did not complete the MBSR training due to a lack of time. The duration of each session was $2.5 \mathrm{~h}$ and comprised sharing and reflecting on past week's experiences, meditation exercises, and presentations providing theoretical insight. Participants were encouraged to practice meditation exercises at home on a daily basis (30-60 min) and implement various smaller mindfulness exercises in their daily routine. Participants in the WLC condition were invited to complete the MBSR training at the end of the study.

\section{Suction blister model}

In order to induce wounds, a standardized procedure for suction blisters was adopted as previously described (Glaser et al. 1999; Kiecolt-Glaser et al. 2005; Zimmerli and Gallin 1987). In short, a trained assistant created eight 8-mm diameter blister wounds. Prior to wound induction a mild depilatory cream was applied to the skin in order to remove hair at the wound site. An acrylic template with eight holes was attached to the disinfected volar surface of the non-dominant forearm at $2 \mathrm{~cm}$ from the elbow joint, 
after which a mild constant $350 \mathrm{mmHg}$ suction with a vacuum pump (Neuro probe, Cabin John, MD, ISA) was applied via the holes until blisters developed (1-1.5 h). During the whole procedure a $250 \mathrm{~W}$ infrared lamp was placed at $30 \mathrm{~cm}$ from the suction site in order to increase the rate of blister formation, simultaneously ensuring that the temperature above the skin did not exceed $37^{\circ} \mathrm{C}$. Next, the epidermis of each blister was removed using a sterile surgical knife, and a plastic sterile 8-well template was attached over the blister wounds. Each well was filled with $1.0 \mathrm{~mL}$ of sterile saline solution $(0.9 \%$ Sodium Chloride, $\mathrm{NaCl}$ ) and sealed off sterile. At 3, 6, and $22 \mathrm{~h}$ after wound induction, wound fluid was taken from the wells with a sterile syringe. Wound fluid samples were centrifuged at $5000 \mathrm{G}$ for $5 \mathrm{~min}$ at $4{ }^{\circ} \mathrm{C}$ in order to obtain undiluted supernatant which was then aliquoted over three samples of $200 \mu \mathrm{L}$ each and stored at $-80^{\circ} \mathrm{C}$. Exudate was taken from three wells at 3 and $6 \mathrm{~h}$ post wound induction and from the remaining two wells at $22 \mathrm{~h}$ post wound induction. The well plate was left in place until the last sample of wound fluid was taken (around 8 AM on the next day), after which the wound site was covered with a large sterile water-resistant bandage. The assistant inducing the wounds was blind to group status. Participants were requested to refrain from strenuous physical exercise, sugar, caffeine, and alcohol consumption, or the use of recreational drugs for $22 \mathrm{~h}$ after wound induction (DeRijk et al. 1997).

\section{Wound healing}

Participants briefly revisited the research unit on day 3, 4, 5, 6, 7, and 10 between $10 \mathrm{AM}$ and $12 \mathrm{AM}$ to monitor the progress of wound healing relating to TEWL and wound surface area measurements.

Trans-epidermal water loss TEWL was used as an index for wound permeability. TEWL measures the evaporation rate in the air layer adjacent to the skin, which increases when the skin is damaged and decreases as the barrier function of the skin recovers (e.g. Altemus et al. 2001). An evaporimeter (VapoMeter, Delfin Technologies, Stamford, CT, USA), was used to determine TEWL above the wound site. Room temperature was maintained at $20-22{ }^{\circ} \mathrm{C}$. Upon arrival participants relaxed for $10 \mathrm{~min}$ to control for any preceding physical exertion. The mean TEWL of two measurements of the two most proximal blisters to the elbow was obtained daily from 3 to 7 days and at 10 days post wound induction in addition to control values measured at two sites on the contralateral arm. The average control values per day were then subtracted from TEWL values above the wound site to control for normal variations in TEWL. Due to unavailability of participants or technical difficulties, 21 participants had available data for each day, 18 for five days, nine for four days, and one for one day.

Wound surface area Wound surface area was determined by taking digital images of the wound site. The participant's arm was positioned at $28 \mathrm{~cm}$ from the camera's lens and built-in camera lights controlled the lighting. For calibration purposes an 8-mm diameter circle sticker was placed next to the wound site. ImageJ software (version 1.44, NIH, Bestheda, MD, USA) was used to map the surface areas of each blister. After calibration of the photograph, the wound area of each blister was assessed in $\mathrm{mm}^{2}$ within a hand-drawn delineated area, including the scab but excluding erythema and scar tissue. Two trained raters blind to the experimental conditions analyzed each photograph. Inter-rater difference was computed for each wound surface area as percent deviation of rater A from rater B. Surface area measurements corresponding to inter-rater differences of $>20 \%$ and an absolute difference of at least $2 \mathrm{~mm}^{2}$ were assessed again by a third rater (rater C, $1.66 \%$ of measurements). Mean wound surface area was calculated by averaging the values of rater $\mathrm{A}$ and $\mathrm{B}$, or in case of a third rater, across the value of rater $\mathrm{C}$ and the value of rater $A$ or $B$ that was closest to rater $C$. A two-way random effect model with absolute agreement

Table 1 Means (SD) of pre- and post-intervention questionnaire scores for both conditions

\begin{tabular}{|c|c|c|c|c|}
\hline & \multicolumn{2}{|l|}{ Pre-intervention } & \multicolumn{2}{|l|}{ Post-intervention } \\
\hline & $\mathrm{WLC}(\mathrm{n}=25)$ & $\operatorname{MBSR}(\mathrm{n}=21)$ & $\mathrm{WLC}(\mathrm{n}=22)$ & $\operatorname{MBSR}(\mathrm{n}=22)$ \\
\hline 1. KIMS & $126(12.4)$ & $121(14.2)$ & $125(13.2)$ & $134(15.5)$ \\
\hline 2. MAAS & $3.77(0.71)$ & $3.63(0.60)$ & $3.68(0.54)$ & $3.87(0.60)$ \\
\hline 3. LOT-R & $21.4(4.05)$ & $20.3(3.86)$ & $22.7(5.31)$ & $22.5(4.13)$ \\
\hline 4. RRQ-rum & $3.54(0.76)$ & $3.71(0.57)$ & $3.50(0.62)$ & $3.24(0.57)$ \\
\hline 5. RRQ-refl & $3.32(0.65)$ & $3.52(0.66)$ & $3.31(0.82)$ & $3.60(0.65)$ \\
\hline
\end{tabular}

WLC waiting-list control group, MBSR mindfulness-based stress reduction group, KIMS Kentucky Inventory of Mindfulness Skills, MAAS Mindfulness Attention Awareness Scale, LOT-R Life Orientation Test Revised, RRQ-rum Rumination-Reflection Questionnaire-rumination subscale, $R R Q$-refl Rumination-Reflection Questionnaire-reflection subscale 
intra-class correlation coefficient (ICC) was then performed to test inter-rater reliability for each time point and blister. All ICC $(2,2)$ values were $>.95$. Due to limited availability of participants and technical difficulties on some days, wound surface area data was incomplete. Surface area data was collected for each day for 18 participants, five days for 20 participants, four days for eight participants, three days for one participant, one day for one participant. No surface area data was available for one participants.

\section{Cytokines and growth factors}

Wound exudate was analysed for IL-1 $\beta$, IL-6, IL-8 and TNF$\alpha$, VEGF, sFlt-1, and PlGF by a multi-array detection system based on electrochemiluminescence technology (MesoScale Discovery SECTOR Imager 2400, Gaithersburg, MD, USA). The intra- and inter-assay coefficients of variation (CVs) were 6.9 and $8.7 \%$ for IL-6, 3.5 and $4.1 \%$ for IL-8, 5.9 and $8.6 \%$ for TNF- $\alpha, 4.7$ and $7.0 \%$ for PIGF, 6.2 and $3.3 \%$ for sFlt-1, and 5.3 and $2.8 \%$ for VEGF, respectively. Because IL-1 $\beta$ was measured using one kit, only an intra-assay $\mathrm{CV}$ of $10.7 \%$ could be computed. Cytokines were measured in the 3,6 , and $22 \mathrm{~h}$ samples, whereas growth factors were only measured in the $22 \mathrm{~h}$ samples. This was because it has been shown that these growth factors only start to increase in concentration approximately 1 day post injury (Johnson and Wilgus 2014). One participant had missing cytokine and growth factor data at 3 and $6 \mathrm{~h}$, and nine participants at $22 \mathrm{~h}$ due to unavailability of the participant or leakage of the well template, leaving 39 participants with data for all three time points.

\section{Procedure}

The KIMS, MAAS, LOT-R, and RRQ were administered four days before the start of the intervention through an online questionnaire program. During the next eight weeks participants followed a MBSR program or started a waiting period. Thereafter, participants completed the same set of questionnaires to assess the effect of the intervention on these variables. Participants were then individually invited to the research unit for the wound induction procedure within 1 month after completion of the MBSR intervention. Participants were asked to continue practicing mindfulness in the intervening period. The day of the wound induction began at $8 \mathrm{AM}$ with the suction blister procedure. During suction which took approximately $75 \mathrm{~min}$, participants were provided with breakfast and filled in some questionnaires (not further reported). Participants remained in the lab until $6 \mathrm{~h}$ had transpired. During this time they performed a few computer tasks and spent the rest of the time doing personal activities (e.g. reading, work). At 3 and
$6 \mathrm{~h}$ post wound induction wound fluid was collected. The next day, participants returned to the lab at 8 AM to provide the final samples of wound exudate and to have the well template removed. Participants briefly revisited the research unit on day 3-7 and day 10 post wound induction between $10 \mathrm{AM}$ and $12 \mathrm{AM}$ to measure TEWL and to have a photograph taken of the blisters. During the intervening periods, the wound site remained covered with sterile adhesive bandage.

\section{Statistical analysis}

All statistical analyses were performed using SPSS 22.0. Descriptive statistics were computed and independent samples $t$ tests and a Chi square test were used to check whether randomization was successful. Two-way analyses of variance (ANOVAs) with condition (WLC vs. MBSR) and sex (men vs. women) as independent variables were performed to check baseline differences in questionnaire scores. Random intercept mixed regression analyses were performed for all outcome measures. Sex was included as factor, as it is known that men and women exhibit distinct physiological properties of the skin (Gilliver et al. 2007). We started testing saturated models including all predictors and their interactions and continued with stepwise exclusion of non-significant interaction terms. Primary outcome measures were TEWL and wound surface area and secondary outcomes were cytokines (IL-1 $\beta$, IL-6, IL-8 and TNF- $\alpha$ ) and growth factors (VEGF, PlGF, and sFlt-1). Statistical significance was assumed to exist at $\alpha \leq .05$ for primary outcomes and at an adjusted $\alpha \leq .01$ for secondary outcomes in order to correct for multiple testing.

Mixed regression analyses with time (pre- vs. post-intervention) as level 1 (within-subjects) factor and condition (WLC vs. MBSR) and sex (men vs. women) as level 2 (between-subjects) factors were conducted in order to test the effect of MBSR on change in KIMS, MAAS, LOT-R, RRQ-reflection, and RRQ-rumination from pre- to postintervention. To examine the effect of MBSR on TEWL, wound surface area, cytokine and growth factor levels, mixed regression analyses with blister (blister 1-8) as level 1 (within-subjects) predictor, time (day 3-7 and 10 post wound induction) as level 2 (within-subjects) predictor, and condition (WLC vs. MBSR) and sex (men vs. women) as level 3 (between-subjects) predictors were performed for TEWL and wound surface area. In these models, blisters were nested within time and time was nested within individuals.

In the models analyzing cytokine data, time $(3,6$, and $22 \mathrm{~h}$ post wound induction) was defined as level 1 (withinsubjects) factor and condition (WLC vs. MBSR) and sex (men vs. women) as level 2 (between-subjects) factors. Only one level was specified for the models analyzing 
growth factors, namely condition (WLC vs. MBSR) and sex (men vs. women) as level 1 (between-subjects) variables.

\section{Results}

\section{Baseline statistics}

Two-way ANOVAs with condition and sex as independent variables revealed no significant differences between the conditions and sexes at baseline in mindfulness, optimism, rumination and reflection (Table 1). Participants in the two conditions neither differed significantly in age $(t(47)=-.50, p=.62)$, nor $\operatorname{sex}\left(\chi^{2}(1)=.34, p=.56\right)$.

\section{Self-report assessment}

Multilevel random intercept modeling was conducted to test the effect of MBSR on change in KIMS, MAAS, LOT$\mathrm{R}$, RRQ-reflection, and RRQ-rumination from pre- to postintervention. A significant time $\times$ condition $\times$ sex interaction for KIMS was found $(F(1,39.5)=8.53, p=.006)$. Testing multilevel models with a time $\times$ condition interaction term for both sexes separately revealed a significant time $\times$ condition interaction for KIMS in women $(F(1$, $34.4)=8.54, p=.006)$ and men $(F(1,5.30)=14.5$, $p=.011)$. Participants in the MBSR condition improved more in terms of KIMS than WLC subjects. This improvement was larger for men than for women. The time $\times$ condition $\times$ sex interaction also reached significance for rumination $(F(1,39.5)=4.79, p=.035)$. Separate multilevel models tested the time $\times$ condition interaction for both sexes and showed a significant time $\times$ condition interaction for rumination in men $(F(1$, $4.95)=7.87, \quad p=.038)$, but not in women $(F(1$, $35.0)=1.99, p=.17)$; rumination decreased more in male participants in the MBSR condition than in the WLC condition. For the MAAS, LOT-R and RRQ-reflection no time $\times$ condition $\times$ sex or time $\times$ condition interactions were detected.

\section{Wound healing}

\section{Trans-epidermal water loss}

None of the interactions with blister were significant in the multilevel models for TEWL and therefore excluded. Because a significant time $\times$ condition $\times$ sex interaction was observed $(F(5,439)=4.86, p<.001)$ separate analyses for women and men were performed. For women no significant time $\times$ condition interaction was found $(F(5$, $382)=.20, p=.96$ ), but it did reach significance for men
$(F(5,59.4)=8.94, p<.001 ;$ Table 2$)$. Exploring the simple effects of condition per time point in men demonstrated a significant effect of condition at day $3(b=104$, $p<.001)$ and day 4 post wound induction $(b=83.3$, $p<.001)$. TEWL was lower in the MBSR than the WLC condition at 3 and 4 days after wounding. Figure 1a displays the estimated marginal means for TEWL across sex.

\section{Wound surface area}

After excluding all non-significant four- and three-way interactions, multilevel modeling revealed a significant blister $\times$ condition $\times$ sex interaction $(F(7,1784)=5.49$, $p<.001)$ and time $\times$ condition $\times$ sex interaction $(F(5$, $1793)=3.72, p=.002$ ) for wound surface area. The significant blister $\times$ condition $\times$ sex interaction was not of interest for the present study, but was retained in the model during subsequent testing. Multilevel models for women and men separately revealed significant time $\times$ condition interaction effects in both women $(F(5,1550)=4.90$, $p<.001)$ and men $(F(5,275)=3.82, p=.002)$. The wound surface area scores as estimated by the final multilevel models are presented in Fig. 1b. For women, the simple effect of condition was not significant on any time point (see Table 2). In men, the simple effect of condition on day 3 was significant ( $b=6.02, p=.010$, see Table 2 ). The simple effects of condition on subsequent days were not significant. ${ }^{1}$

\section{Biological markers}

Table S1 displays the absolute cytokine and growth factor concentrations as determined by the multi-array platform across condition and sex (Supplemental Material).

\section{Pro-inflammatory cytokines}

The time $\times$ condition $\times$ sex interaction did not reach significance for any of the cytokines. Consequently, these interaction terms were excluded from the multilevel models. For IL- 8 , the time $\times$ condition interaction $(F(2$, $286)=5.96, p=.003)$ and time $\times$ sex interaction $(F(2$, $292)=21.8, p<.001)$ were significant. Simple effect analyses per time point revealed significant effects at $22 \mathrm{~h}$ post wound induction for condition $(b=1014, p<.001)$ and sex $(b=3059, p<.001)$. Levels of IL-8 were higher in the WLC group relative to the MBSR group and higher in men compared to women (see Fig. 2). For IL-1 $\beta$, IL-6 and TNF- $\alpha$, only the time $\times$ sex interactions reached significance (IL-1 $\beta$ : $F(2,290)=31.4, p<.001$; IL-6: $F(2$,

\footnotetext{
${ }^{1}$ All wounds healed normally but five participants experienced postinflammatory hyperpigmentation for longer than 6 months.
} 
Fig. 1 Estimated marginal means for trans-epidermal water loss (a) and wound surface area scores (b) for men and women. WLC $=$ waiting-list control group; MBSR = MindfulnessBased Stress Reduction group; $\mathrm{EMM}=$ estimated marginal means; TEWL $=$ transepidermal water loss

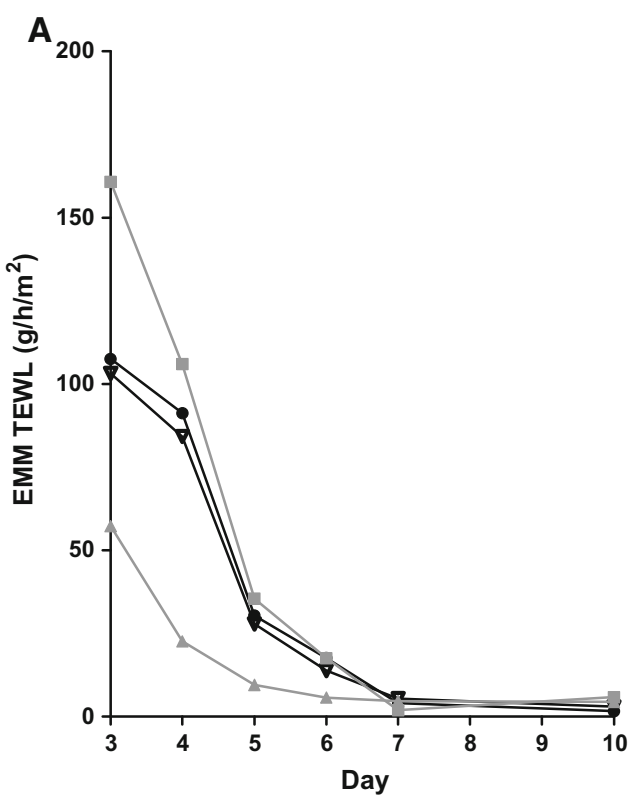

$291)=7.19, \quad p=.001 ; \quad$ TNF- $\alpha: \quad F(2, \quad 299)=27.0$, $p<.001)$. At $22 \mathrm{~h}$ after wounding, men displayed higher levels of IL-1 $\beta(b=3751, p<.001)$, IL-6 $(b=3369$, $p<.001)$ and TNF- $\alpha(b=3684, p<.001)$ than women.

\section{Growth factors}

For PlGF a significant main effect of condition $(F(1$, $36)=7.38, p=.010$ ) was found. At $22 \mathrm{~h}$ post wound induction the WLC group exhibited higher levels of PIGF relative to the mindfulness group ( $b=7.74, p=.010$ ) (see Fig. 2). A significant condition $\times$ sex interaction was observed for $\operatorname{VEGF}(F(1,37.6)=15.5, p<.001)$ and sFlt$1(F(1,36.7)=15.6, p<.001)$. Simple effect analyses showed that the effect of condition was only significant for men $22 \mathrm{~h}$ after injury (VEGF: $b=2617, p<.001$; sFlt-1: $b=4002, p<.001$ ) with participants in the mindfulness condition displaying lower levels of both VEGF and sFlt-1 than participants in the WLC condition.

\section{Post hoc analyses}

Because not everyone profited from the MBSR intervention to the same degree, we examined post hoc whether the increase in mindfulness levels after the intervention was related to TEWL, wound surface area, cytokine and growth factor levels. Similar multilevel models were built with the increase in mindfulness (diffKIMS) as predictor instead of condition. For TEWL, a significant time $\times$ diffKIMS interaction was found $(F(5,399)=10.45, p<.001)$. The effect of diffKIMS on day $3(b=-1.86, p<.001)$ and day 4 after wound induction $(b=-2.11, p<.001)$ was significant, showing that larger increases in mindfulness

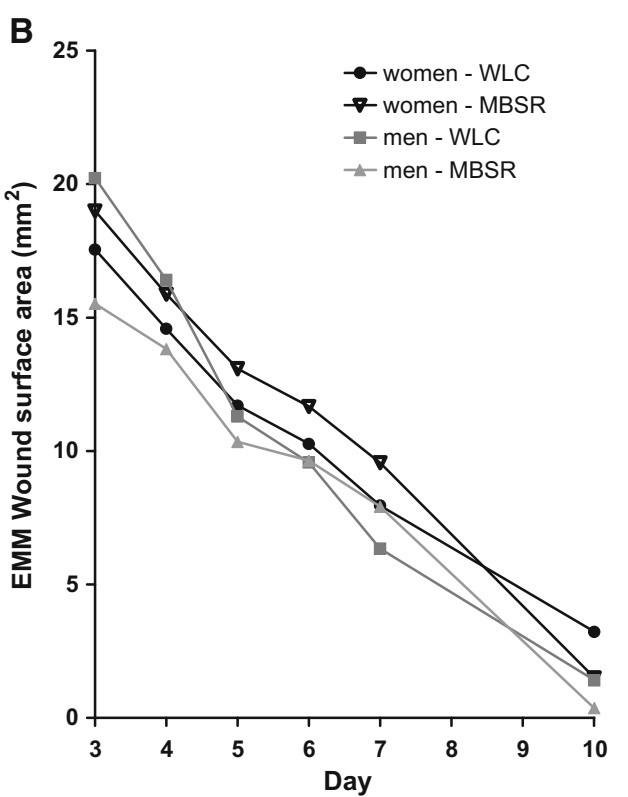

were related to greater decreases in TEWL, irrespective of sex (see Fig. 3). When the analysis was repeated for women only, this also revealed a significant time $x$ diffKIMS interaction $(F(5,332)=6.36, p<.001)$. The effect of diffKIMS was significant at day $3(b=-1.46$, $p=0.004)$ and at day 4 after injury $(b=-2.45$, $p<.001)$. For wound surface area, a significant time $\times-$ diffKIMS $\times$ sex interaction was found $(F(5$, $1589)=4.80, p<.001)$. However, simple effect analyses per time point did not reveal any significant diffKIMS effects for men or women.

The post hoc analyses also indicated significant time $\times$ diffKIMS interactions for IL- $1 \beta(F(2,252)=5.34$, $p=.005)$ and IL-8 $(F(2,254)=7.31, p=.001)$. Larger increases in mindfulness were associated with lower levels of IL-1 $\beta(b=-46.0, p<.001)$ and IL-8 $(b=-51.7$, $p<.001)$ at $22 \mathrm{~h}$ after wounding. The diffKIMS $\times \operatorname{sex}$ interaction was significant for $\operatorname{VEGF}(F(1,38.1)=9.19$, $p=.004)$ and sFlt-1 $(F(1,33.6)=7.86, p=.008)$, with higher levels of diffKIMS related to lower levels of VEGF $(b=-75.6, \quad p=.001) \quad$ and sFlt-1 $\quad(b=-118.6$, $p=.001)$ in men at $22 \mathrm{~h}$ after wounding.

\section{Discussion}

This study examined the effect of an 8-week MBSR intervention on wound healing. Because our analyses indicated an interaction of condition with sex, separate analyses for men and women were performed. Although for men significant effects on TEWL and wound size seemed to be present, we cannot reliably interpret these findings as only three men had received the MBSR inter- 

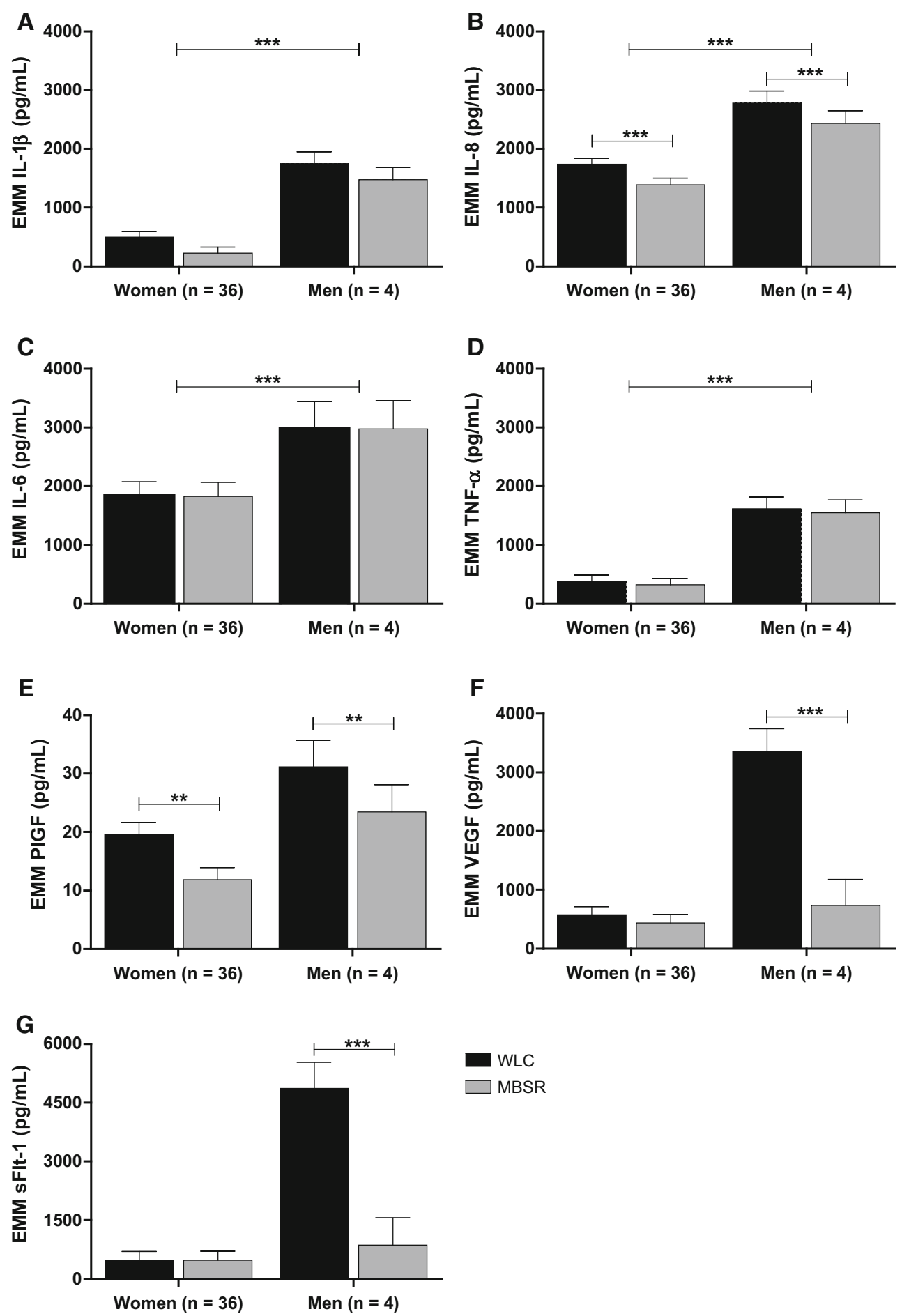

Fig. 2 Estimated marginal means and standard error of means for IL$1 \beta$ (a), IL-8 (b), IL-6 (c), TNF- $\alpha$ (d), PlGF (e), VEGF (f), and sFlt-1 (g) at $22 \mathrm{~h}$ post wound induction. WLC $=$ waiting-list control group; MBSR $=$ Mindfulness-Based Stress Reduction group; EMM = esti-

vention. For women, no differences in wound healing were found between participants in the MBSR and WLC condition; there were no effects on TEWL, and although a significant time $\times$ condition effect was found for wound

mated marginal means; IL $=$ interleukin; TNF- $\alpha=$ tumor necrosis factor $\alpha ; \quad \mathrm{PIGF}=$ placental growth factor; $\mathrm{VEGF}=$ vascular endothelial growth factor; sFlt-1 = soluble Fms-like tyrosine kinase-1. $* * p \leq .01 ; * * p \leq .001$

surface area, none of the single time points significantly differed between the conditions. Thus, the hypothesis that receiving a MBSR training would lead to faster wound healing was not supported. 

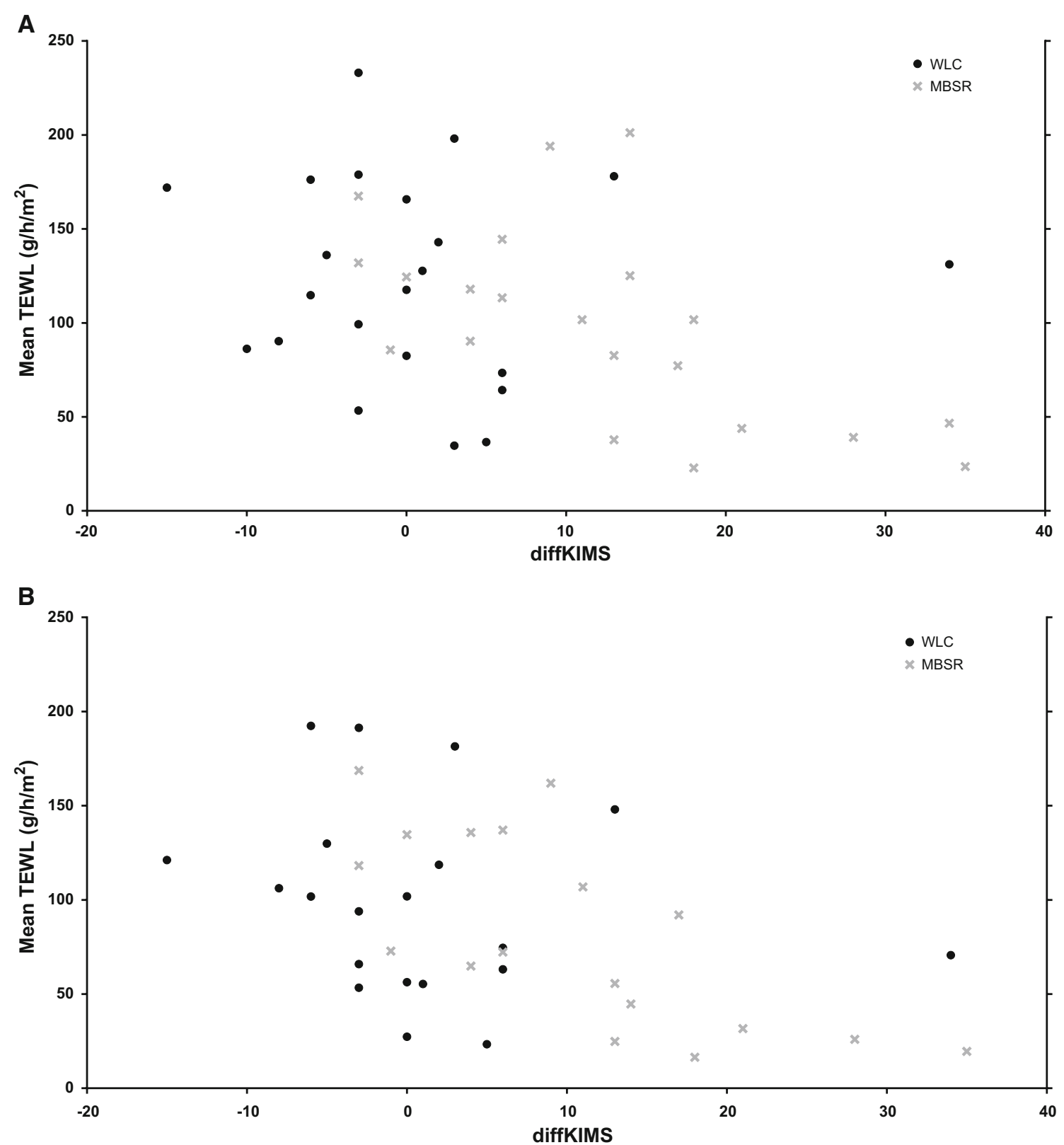

Fig. 3 Scatterplots displaying the relationship between mean TEWL scores and diffKIMS for the WLC and MBSR group at day 3 (a) and day 4 (b) post wound induction. WLC $=$ waiting-list control group;

Since not everybody benefited to the same extent of the MBSR intervention, we examined, post hoc, whether the actual improvement in mindfulness was predictive for the speed of wound healing. This analysis showed that larger increases in mindfulness scores were related to greater decreases in TEWL, but not wound size, at day 3 and 4 . Because men showed the largest increases in mindfulness after MBSR this may possibly have contributed to the fact that the condition effect on TEWL and wound size was found to be significant in men. Nevertheless, the association between the actual change in mindfulness and speed of
MBSR = Mindfulness-Based Stress Reduction group; TEWL = trans-epidermal water loss; diffKIMS $=$ amount of increase in mindfulness levels after the MBSR intervention

wound healing was also apparent when analyses were repeated for women only. The finding that the participants who improved most in mindfulness skills benefitted most in terms of wound healing is consistent with a study demonstrating that the amount of practice completed by the participants in the MBSR condition determined its effect on a healing-related process. Specifically, Rosenkranz et al. (2013) found that total time practicing MBSR was negatively related to TNF- $\alpha$ level in skin blisters in response to psychological stress and capsaicin exposure. We did not measure practicing time but it could be hypothesized that 
Table 2 Parameter estimates for the final multilevel models for trans-epidermal water loss and wound surface area across women and men

\begin{tabular}{|c|c|c|c|c|c|c|c|c|c|c|c|c|c|c|c|c|}
\hline \multirow[b]{3}{*}{ Simple effects } & \multicolumn{8}{|l|}{ TEWL } & \multicolumn{8}{|c|}{ Wound surface area } \\
\hline & \multicolumn{4}{|c|}{ Women $(n=41)$} & \multicolumn{4}{|c|}{ Men $(n=8)$} & \multicolumn{4}{|c|}{ Women $(n=40)$} & \multicolumn{4}{|c|}{ Men $(n=8)$} \\
\hline & $\mathrm{b}$ & SE & $t$ & $p$ & $\mathrm{~b}$ & $\mathrm{SE}$ & $t$ & $p$ & $\mathrm{~b}$ & SE & $t$ & $p$ & $\mathrm{~b}$ & SE & $t$ & $p$ \\
\hline $\begin{array}{l}\text { Condition effect on } \\
\text { day } 3\end{array}$ & 4.37 & 9.29 & 0.47 & .64 & 104 & 15.23 & 6.80 & $<.001$ & -1.20 & 1.54 & -0.78 & .44 & 6.02 & 2.16 & 2.78 & .010 \\
\hline $\begin{array}{l}\text { Condition effect on } \\
\text { day } 4\end{array}$ & 7.02 & 9.61 & 0.73 & .47 & 83.3 & 21.9 & 3.80 & $<.001$ & -1.03 & 1.54 & -0.67 & .51 & 3.89 & 2.51 & 1.55 & .13 \\
\hline $\begin{array}{l}\text { Condition effect on } \\
\text { day } 5\end{array}$ & 2.52 & 9.78 & 0.26 & .80 & 25.9 & 18.0 & 1.44 & .16 & -1.12 & 1.56 & -0.71 & .48 & 2.25 & 2.30 & 0.98 & .34 \\
\hline $\begin{array}{l}\text { Condition effect on } \\
\text { day } 6\end{array}$ & 3.84 & 9.98 & 0.39 & .70 & 11.9 & 16.2 & .73 & .47 & -1.14 & 1.56 & -0.73 & .47 & 1.24 & 2.21 & 0.56 & .58 \\
\hline $\begin{array}{l}\text { Condition effect on } \\
\text { day } 7\end{array}$ & -1.34 & 9.70 & -0.14 & .89 & -2.81 & 15.2 & -0.18 & .86 & -1.33 & 1.55 & -0.86 & .39 & -0.27 & 2.16 & -0.12 & .90 \\
\hline $\begin{array}{l}\text { Condition effect on } \\
\text { day } 10\end{array}$ & -1.35 & 10.0 & -0.14 & .89 & 1.37 & 14.6 & 0.094 & .93 & 1.98 & 1.56 & 1.27 & .21 & 2.34 & 2.12 & 1.10 & .28 \\
\hline
\end{tabular}

$b$ Estimate, $S E$ standard error, $T E W L$ trans-epidermal water loss

those who practice most also gain the most benefit from the intervention.

This study adds to current knowledge by investigating the role of local pro-inflammatory cytokine and growth factor release as potential underlying mechanisms of improved wound healing. We found that compared to WLC participants, both men and women in the MBSR condition had lower levels of IL-8, and PIGF at the wound site $22 \mathrm{~h}$ after wounding. Post hoc analyses also showed that greater increases in mindfulness were related to larger decreases in IL-1 $\beta$ and IL-8 $22 \mathrm{~h}$ after wounding. The finding that proinflammatory cytokine levels in wound fluid are lower in participants in the MBSR condition is consistent with the results of one earlier study on the effect of MBSR on local cytokine expression (Rosenkranz et al. 2013). However, the result is contrary to studies that found that cytokine levels were suppressed by stress (Broadbent et al. 2003; Glaser et al. 1999; Kiecolt-Glaser et al. 2005). It is probably an oversimplification to assume that an increased inflammatory response in the early phase of healing is always beneficial. For example, elevated inflammatory responses can actually impede healing, as illustrated by the finding of heightened cytokine levels in chronic wounds (Beidler et al. 2009). An adequate balance between proand anti-inflammatory forces is quintessential for optimal wound healing (e.g. Eming et al. 2007). Whether increases or decreases in cytokines are beneficial, may among other issues, depend on the base level within a certain individual. It should be borne in mind that we studied a healthy sample, not specifically selected for elevated stress level and/or compromised wound healing. In these participants decreased local cytokine production might be adaptive, especially in case of small blister wounds, as a greater inflammatory response may impede the subsequent prolif- eration and remodeling phase and thereby the overall healing process (Mustoe et al. 2006). Further, since only limited tissue destruction is present in suction wounds and microbial contamination is minimal, there may be little need for a pro-inflammatory response. Future research would therefore benefit from examining subjects selected for elevated stress levels to investigate whether MBSR could diminish stress-induced delays in wound healing.

Growth factors, such as VEGF and PlGF, play an essential role in wound healing by performing multiple functions such as stimulating angiogenesis and the proliferation of fibroblasts and endothelial cells. Growth factors are expressed at low levels in the skin, but start to increase at about one day after injury (Johnson and Wilgus 2014). VEGF and PIGF levels were clearly detectable $22 \mathrm{~h}$ after blistering. Contrary to expectations, MBSR was associated with lower levels of PIGF in wound fluid after $22 \mathrm{~h}$. This finding is puzzling given previous research showing the importance of an up-regulation of PIGF for wound angiogenesis and thereby tissue repair. For instance, a deficiency in angiogenesis has been demonstrated to cause compromised wound healing in PlGF knock-out mice (Carmeliet et al. 2001). The functional significance of growth factors depends on the availability of receptors which they can bind to. The VEGF receptor family consists of three types of which one is important for wound healing and also binds PlGF: Fms-like tyrosine kinase-1 (Flt-1). There exist two forms of this receptor, namely one functional full length receptor (Flt-1) and a soluble decoy receptor (sFlt-1) that suppresses both VEGF and PlGF activity upon binding. The balance between the availability of the full length and the soluble receptor determines the net effect of VEGF and PlGF on wound healing (Shibuya 2015). Although we measured the level of sFlt-1, we did not assess availability 
of Flt-1, making it difficult to draw conclusions about the effects of decreases in PIGF on wound healing. It therefore remains unclear what the exact role of PlGF is as a potential mediator of MBSR on wound healing.

Some limitations of this study should be mentioned. First, only few male participants were included and the numbers were even smaller for cytokine and growth factor data due to missing data. Further, although we did not include a measure of perceived stress, we did assess rumination at pre- and post-intervention and found that MBSR significantly reduced rumination levels in men. Men also scored highest on rumination before the intervention. When taking rumination as a proxy for stress susceptibility, MBSR seemed to have benefited participants most in need of stress reduction. Indeed, post hoc examination revealed that larger decreases in rumination from pre- to post-intervention were associated with greater decreases in TEWL at day 3 and 4 in men. In addition, in the overall sample the effect of condition on TEWL was mediated by rumination (data not shown). Possibly, when selecting participants for high stress and rumination levels, we may observe an overall MBSR effect on wound healing. Therefore, research is warranted to further elucidate whether the effect of MBSR is mediated by reduced stress levels. Another limitation was that it was not possible to monitor wound size and TEWL from the moment of wound induction onwards, due to the attached well plate that was used for collecting wound fluid. It would have been valuable to examine healing from the moment of wound induction onwards to study early stages of healing. Additionally, although interrater reliability was excellent, assessment of wound surface area using digital images is not without problems. Determining the margin of the wounds is sometimes difficult, especially during later days when scabs start to develop. Moreover, photography only assesses healing at the surface level, whereas areas deep within the wound are equally important for the healing process (Dyson et al. 2003). However, TEWL was our primary outcome and there was good concordance between these assessments. Lastly, the experimental wounds were small and future research into the clinical effects of MBSR on wound healing would benefit from examining various degrees of surgical and chronic wounds.

To conclude, the current outcomes provide preliminary evidence that MBSR can affect the early stages of wound healing. To our knowledge, this study was the first to examine the effects of a psychological intervention on the levels of growth factors in wound fluid and should therefore be replicated and extended to other relevant factors. Future research into the exact mechanisms underlying improved wound healing by psychological factors is highly warranted.
Funding This study was funded by an Innovative Research Grant (VICI) from the Netherlands Organization of Scientific Research that has been awarded to M. Peters (Grant Number 453-07-005).

\section{Compliance with ethical standards}

Conflict of interest Astrid Meesters, Yvo M. C. In den BoschMeevissen, Chantal A. H. Weijzen, Wim A. Buurman, Mario Losen, Jan Schepers, Monique R. T. M. Thissen, Hugo J. E. M. Alberts, Casper G. Schalkwijk, and Madelon L. Peters declare that they have no conflict of interest.

Human and animal rights and Informed consent All procedures followed were in accordance with ethical standards of the responsible committee on human experimentation (institutional and national) and with the Helsinki Declaration of 1975, as revised in 2000. Informed consent was obtained from all patients for being included in the study.

Open Access This article is distributed under the terms of the Creative Commons Attribution 4.0 International License (http:// creativecommons.org/licenses/by/4.0/), which permits unrestricted use, distribution, and reproduction in any medium, provided you give appropriate credit to the original author(s) and the source, provide a link to the Creative Commons license, and indicate if changes were made.

\section{References}

Altemus, M., Rao, B., Dhabhar, F. S., Ding, W., \& Granstein, R. D. (2001). Stress-induced changes in skin barrier function in healthy women. Journal of Investigative Dermatology, 117, 309-317. https://doi.org/10.1046/j.1523-1747.2001.01373.x

Baer, R. A., Smith, G. T., \& Allen, K. B. (2004). Assessment of mindfulness by self-report: The Kentucky inventory of mindfulness skills. Assessment, 11, 191-206. https://doi.org/10.1177/ 1073191104268029

Beidler, S. K., Douillet, C. D., Berndt, D. F., Keagy, B. A., Rich, P. B., \& Marston, W. A. (2009). Inflammatory cytokine levels in chronic venous insufficiency ulcer tissue before and after compression therapy. Journal of Vascular Surgery, 49, 1013-1020. https://doi.org/10.1016/j.jvs.2008.11.049

Bosch, J. A., Engeland, C. G., Cacioppo, J. T., \& Marucha, P. T. (2007). Depressive symptoms predict mucosal wound healing. Psychosomatic Medicine, 69, 597-605. https://doi.org/10.1097/ PSY.0b013e318148c682

Broadbent, E., Kahokehr, A., Booth, R. J., Thomas, J., Windsor, J. A., Buchanan, C. M., et al. (2012). A brief relaxation intervention reduces stress and improves surgical wound healing response: $\mathrm{A}$ randomised trial. Brain, Behavior, and Immunity, 26, 212-217. https://doi.org/10.1016/j.bbi.2011.06.014

Broadbent, E., \& Koschwanez, H. E. (2012). The psychology of wound healing. Current Opinion in Psychiatry, 25, 135-140. https://doi.org/10.1097/YCO.0b013e32834e1424

Broadbent, E., Petrie, K. J., Alley, P. G., \& Booth, R. J. (2003). Psychological stress impairs early wound repair following surgery. Psychosomatic Medicine, 65, 865-869. https://doi.org/ 10.1097/01.PSY.0000088589.92699.30

Brown, K. W., \& Ryan, R. M. (2003). The benefits of being present: Mindfulness and its role in psychological well-being. Journal of Personality and Social Psychology, 84, 822-848. https://doi.org/ 10.1037/0022-3514.84.4.822 
Carmeliet, P., Moons, L., Luttun, A., Vincenti, V., Compernolle, V., De Mol, M., et al. (2001). Synergism between vascular endothelial growth factor and placental growth factor contributes to angiogenesis and plasma extravasation in pathological conditions. Nature Medicine, 7, 575-583. https://doi.org/10.1038/ 87904

Christian, L. M., Graham, J. E., Padgett, D. A., Glaser, R., \& KiecoltGlaser, J. K. (2006). Stress and wound healing. NeuroImmunoModulation, 13, 337-346. https://doi.org/10.1159/ 000104862

Cole-King, A., \& Harding, K. G. (2001). Psychological factors and delayed healing in chronic wounds. Psychosomatic Medicine, 63, 216-220. https://doi.org/10.1097/00006842-20010300000004

DeRijk, R., Michelson, D., Karp, B., Petrides, J., Galliven, E., Deuster, P., et al. (1997). Exercise and circadian rhythm-induced variations in plasma cortisol differentially regulate interleukin-1 beta (IL-1 beta), IL-6, and tumor necrosis factor-alpha (TNF alpha) production in humans: High sensitivity of TNF alpha and resistance of IL-6. Journal of Clinical Endocrinology and Metabolism, 82, 2182-2191. https://doi.org/10.1210/jcem.82.7. 4041

Dyson, M., Moodley, S., Verjee, L., Verling, W., Weinman, J., \& Wilson, P. (2003). Wound healing assessment using $20 \mathrm{MHz}$ ultrasound and photography. Skin Research and Technology, 9, 116-121. https://doi.org/10.1034/j.1600-0846.2003.00020.x

Ebrecht, M., Hextall, J., Kirtley, L. G., Taylor, A., Dyson, M., \& Weinman, J. (2004). Perceived stress and cortisol levels predict speed of wound healing in healthy male adults. Psychoneuroendocrinology, 29, 798-809. https://doi.org/10.1016/s03064530(03)00144-6

Eming, S. A., Krieg, T., \& Davidson, J. M. (2007). Inflammation in wound repair: Molecular and cellular mechanisms. Journal of Investigative Dermatology, 127, 514-525. https://doi.org/10. 1038/sj.jid.5700701

Failla, C. M., Odorisio, T., Cianfarani, F., Schietroma, C., Puddu, P., \& Zambruno, G. (2000). Placenta growth factor is induced in human keratinocytes during wound healing. Journal of Investigative Dermatology, 115, 388-395. https://doi.org/10.1046/j. 1523-1747.2000.00085.x

Garg, A., Chren, M. M., Sands, L. P., Matsui, M. S., Marenus, K. D., Feingold, K. R., et al. (2001). Psychological stress perturbs epidermal permeability barrier homeostasis: Implications for the pathogenesis of stress-associated skin disorders. Archives of Dermatology, 137, 53-59. https://doi.org/10.1001/archderm.137. 1.53

Gilliver, S. C., Ashworth, J. J., \& Ashcroft, G. S. (2007). The hormonal regulation of cutaneous wound healing. Clinics in Dermatology, 25, 56-62. https://doi.org/10.1016/j.clindermatol. 2006.09.012

Glaser, R., Kiecolt-Glaser, J. K., Marucha, P. T., MacCallum, R. C., Laskowski, B. F., \& Malarkey, W. B. (1999). Stress-related changes in proinflammatory cytokine production in wounds. Archives of General Psychiatry, 56, 450-456. https://doi.org/10. 1001/archpsyc.56.5.450

Gouin, J. P., \& Kiecolt-Glaser, J. K. (2011). The impact of psychological stress on wound healing: Methods and mechanisms. Immunology and Allergy Clinics of North America, 31, 81-93. https://doi.org/10.1016/j.iac.2010.09.010

House, S. L. (2015). Psychological distress and its impact on wound healing: An integrative review. Journal of Wound, Ostomy and Continence Nursing, 42, 38-41. https://doi.org/10.1097/won. 0000000000000080

Johnson, K. E., \& Wilgus, T. A. (2014). Vascular endothelial growth factor and angiogenesis in the regulation of cutaneous wound repair. Advances in Wound Care, 3, 647-661. https://doi.org/10. 1089/wound.2013.0517

Kabat-Zinn, J. (1982). An outpatient program in behavioral medicine for chronic pain patients based on the practice of mindfulness meditation: Theoretical considerations and preliminary results. General Hospital Psychiatry, 4, 33-47.

Kabat-Zinn, J. (1990). Full catastrophe living. New York: Delacorte.

Khoury, B., Sharma, M., Rush, S. E., \& Fournier, C. (2015). Mindfulness-based stress reduction for healthy individuals: A meta-analysis. Journal of Psychosomatic Research, 78, 519-528. https://doi.org/10.1016/j.jpsychores.2015.03.009

Kiecolt-Glaser, J. K., Loving, T. J., Stowell, J. R., Malarkey, W. B., Lemeshow, S., Dickinson, S. L., et al. (2005). Hostile marital interactions, proinflammatory cytokine production, and wound healing. Archives of General Psychiatry, 62, 1377-1384. https:// doi.org/10.1001/archpsyc.62.12.1377

Koschwanez, H. E., Kerse, N., Darragh, M., Jarrett, P., Booth, R. J., \& Broadbent, E. (2013). Expressive writing and wound healing in older adults: A randomized controlled trial. Psychosomatic Medicine, 75, 581-590. https://doi.org/10.1097/PSY. 0b013e31829b7b2e

Lowry, S. F. (1993). Cytokine mediators of immunity and inflammation. Archives of Surgery, 128, 1235-1241. https://doi.org/10. 1001/archsurg. 1993.01420230063010

Maple, H., Chilcot, J., Lee, V., Simmonds, S., Weinman, J., \& Mamode, N. (2015). Stress predicts the trajectory of wound healing in living kidney donors as measured by high-resolution ultrasound. Brain, Behavior, and Immunity, 43, 19-26. https:// doi.org/10.1016/j.bbi.2014.06.012

Mustoe, T. A., O'Shaughnessy, K., \& Kloeters, O. (2006). Chronic wound pathogenesis and current treatment strategies: A unifying hypothesis. Plastic and Reconstructive Surgery, 117, 35S-41S. https://doi.org/10.1097/01.prs.0000225431.63010.1b

Nauck, M., Karakiulakis, G., Perruchoud, A. P., Papakonstantinou, E., \& Roth, M. (1998). Corticosteroids inhibit the expression of the vascular endothelial growth factor gene in human vascular smooth muscle cells. European Journal of Pharmacology, 341, 309-315. https://doi.org/10.1016/S0014-2999(97)01464-7

Pyter, L. M., Yang, L., da Rocha, J. M., \& Engeland, C. G. (2014). The effects of social isolation on wound healing mechanisms in female mice. Physiology \& Behavior, 127, 64-70. https://doi. org/10.1016/j.physbeh.2014.01.008

Robinson, H., Jarrett, P., \& Broadbent, E. (2015). The effects of relaxation before or after skin damage on skin barrier recovery: A preliminary study. Psychosomatic Medicine, 77, 844-852. https://doi.org/10.1097/psy.0000000000000222

Robinson, H., Jarrett, P., Vedhara, K., \& Broadbent, E. (2016). The effects of expressive writing before or after punch biopsy on wound healing. Brain, Behavior, and Immunity. https://doi.org/ 10.1016/j.bbi.2016.11.025

Rosenkranz, M. A., Davidson, R. J., Maccoon, D. G., Sheridan, J. F., Kalin, N. H., \& Lutz, A. (2013). A comparison of mindfulnessbased stress reduction and an active control in modulation of neurogenic inflammation. Brain, Behavior, and Immunity, 27, 174-184. https://doi.org/10.1016/j.bbi.2012.10.013

Scheier, M. F., Carver, C. S., \& Bridges, M. W. (1994). Distinguishing optimism from neuroticism (and trait anxiety, self-mastery, and self-esteem): A reevaluation of the Life Orientation Test. Journal of Personality and Social Psychology, 67, 1063-1078. https://doi.org/10.1037//0022-3514.67.6.1063

Segal, Z. V., Williams, J. M. G., \& Teasdale, J. D. (2002). Mindfulness-based cognitive therapy for depression. New York, London: Guilford Press.

Shibuya, M. (2015). VEGF-VEGFR system as a target for suppressing inflammation and other diseases. Endocrine, Metabolic \& 
Immune Disorders: Drug Targets, 15, 135-144. https://doi.org/ 10.2174/1871530315666150316121956

Trapnell, P. D., \& Campbell, J. D. (1999). Private self-consciousness and the five-factor model of personality: Distinguishing rumination from reflection. Journal of Personality and Social Psychology, 76, 284-304. https://doi.org/10.1037/0022-3514. 76.2.284

Walburn, J., Vedhara, K., Hankins, M., Rixon, L., \& Weinman, J. (2009). Psychological stress and wound healing in humans: A systematic review and meta-analysis. Journal of Psychosomatic Research, 67, 253-271. https://doi.org/10.1016/j.jpsychores. 2009.04.002

Weinman, J., Ebrecht, M., Scott, S., Walburn, J., \& Dyson, M. (2008). Enhanced wound healing after emotional disclosure intervention.
British Journal of Health Psychology, 13, 95-102. https://doi. org/10.1348/135910707x251207

Werner, S., \& Grose, R. (2003). Regulation of wound healing by growth factors and cytokines. Physiological Reviews, 83, 835-870. https://doi.org/10.1152/physrev.00031.2002

Zimmerli, W., \& Gallin, J. I. (1987). Monocytes accumulate on Rebuck skin window coverslips but not in skin chamber fluid. A comparative evaluation of two in vivo migration models. Journal of Immunological Methods, 96, 11-17. https://doi.org/10.1016/ 0022-1759(87)90361-9 